$03,09,12$

\title{
Оптическая характеризация структурных и электрических свойств нанослоев $n$-GaP, выращенных на проводящих подложках (001) $n$-GaP
}

\author{
(C) Б.Х. Байрамов ${ }^{1}$, В.В. Топоров ${ }^{1}$, Ф.Б. Байрамов ${ }^{1}$ \\ ${ }^{1}$ Физико-технический институт им. А.Ф. Иоффре РАН, \\ Санкт-Петербург, Россия \\ ${ }^{2}$ Санкт-Петербургский национальный исследовательский академический университет РАН им. Ж.И. Алфёрова, \\ Санкт-Петербург, Россия \\ E-mail: bairamov@mail.ioffe.ru
}

Поступила в Редакцию 10 сентября 2020 г.

В окончательной редакции 10 сентября 2020 г.

Принята к публикации 16 сентября 2020 г.

Сообщается о результатах исследования структурных и электрических свойств гомоэпитаксиального наномасштабного слоя (001) $n$ - GaP, толщиной $70 \mathrm{~nm}$, выращенного методом газофазной эпитаксии из металлоорганических соединений на проводящей сильнолегированной подложке кристалла $n$-GaP, ориентированного по оси (001). В спектре рамановского рассеяния света такого нанослоя $n$-GaP в образце $n$-GaP $/ n-\mathrm{GaP}$ (001) в сравнении со спектром высокоомного кристаллического образца (001) si-GaP удалось обнаружить две достаточно узкие линии, приписанные колебаниям поперечных $\mathrm{TO}(\Gamma)_{-}$фононов и высокочастотным продольным связанным плазмон-фононным $\mathrm{LO}(\Gamma)_{+}$колебаниям. При этом установлено, что спектральные параметры $\mathrm{LO}(\Gamma)_{+}$колебаний как в нанослое $n$-GaP, так и в подложке $(001) n$-GaP существенно отличаются между собой, а также и от спектральных параметров линии продольных оптических LO(Г)_ фононов. Анализ выявленных строгих количественных особенностей спектральных параметров позволил получить ценную информацию о совершенстве кристаллической структуры наномасштабного гомоэпитаксиального слоя (001) n-GaP. Помимо этого, показано что, численные расчеты на основе микроскопической модели рассеяния света $\mathrm{LO}(\Gamma)_{+}$колебаниями, обусловленного механизмами деформационного потенциала и электрооптическим рассеянием, позволили бесконтактным и неразрушающим образом определить онцентрацию $n$ и подвижность $\mu$ свободных носителей заряда гомоэпитаксиального слоя наномасштабной толщины и проводящей сильнолегированной подложки. Полученные значения оказались $n_{\mathrm{hepi}}=(3.25 \pm 0.1) \cdot 10^{17} \mathrm{~cm}^{-3}$ и подвижности $\mu_{\text {hepi }}=(40.0 \pm 0.1) \mathrm{cm}^{2} \cdot \mathrm{V}^{-1} \cdot \mathrm{s}^{-1}$ для гомоэпитаксиального слоя $(001) n$-GaP в сравнении с $n_{\text {subs }}=(2.52 \pm 0.1) \cdot 10^{17} \mathrm{~cm}^{-3}$ и $\mu_{\text {subs }}=(51.0 \pm 0.1) \mathrm{cm}^{2} \cdot \mathrm{V}^{-1} \cdot \mathrm{s}^{-1}$ для подложки $(001) n$-GaP образца $n-\mathrm{GaP} / n-\mathrm{GaP}(001)$.

Ключевые слова: гомоэпитаксиальный наномасштабный слой (001) n-GaP, сильнолегированная подложка, концентрация и подвижность носителей заряда.

DOI: 10.21883/FTT.2021.01.50402.193

\section{1. Введение}

В настоящее время наметилась определенная тенденция к росту выращивания высококачественных ориентированных гомоэпитаксиальных слоев (включая и наномасштабной толщины) широкозонных полупроводников на исходных сильнолегированных пластинах того же материала необходимых для создания приборных структур, демонстрирующих в сравнении с гетероэпитаксиальными слоями лучшую совокупность физических свойств: степени кристаллического совершенства структуры с меньшей плотностью дислокаций и других структурных дефектов, оптических, электрических и иных свойств. Совершенные и высококачественные слои получаются только при условиях послойного роста, происходящего через зарождение на поверхности и рост двумерных островков новой фазы, рассматриваемого как 2D-фазовый переход первого рода, и их последующий рост до образования полного монослоя растущего монокристалла [1-9]. Такие ключевые достоинства гомоэпитаксиальных слоев вместе с тем требуют также развития и максимально эффективных методов исследования их кристаллической структуры и диагностики электрических свойств. Одной из основных проблем технологии приборов на основе гомоэпитаксиальных слоев, выращенных на сильнолегированных подложках являются очевидные сложности определения степени совершенства кристаллической структуры, а также концентрации и подвижности свободных носителей заряда на основе традиционного метода исследования электрических свойств полупроводниковых материалов путем времязатратных процессов предварительного приготовления металлических омических контактов и проведения электрических измерений проводимости и эффекта Холла $[5,7,9]$.

Оптическая спектроскопия рамановского рассеяния света является одним из наиболее привлекательных ме- 
тодов исследования кристаллической структуры и динамики элементарных решеточных и электронных возбуждений. Исчерпывающую информацию о теоретических и экспериментальных исследованиях в этом направлении можно найти в работах [10-24]. В спектрах рамановского рассеяния легированных полупроводников вследствие кулоновского взаимодействия могут проявляться два типа элементарных возбуждений - одночастичные и коллективные. Спектры одночастичного рассеяния света, обусловленные различными флуктуациями плотности электронного газа вследствие наличия зависимости энергии блоховских электронов от квазиимпульса, т. е. непараболичности зон, а также спин-орбитального взаимодействия. При этом, радиус экранирования $r_{s}$ настолько велик, что электронное возбуждение со световым волновым вектором $q$ не экранируется $-q r_{s} \gg 1$ и наиболее существенны флуктуации зарядовой плотности. Спектр квазиупругого электронного рассеяния простирается до $100-200 \mathrm{~cm}^{-1}$ по обе стороны от возбуждающей линии лазера [13-16]. Помимо этого, в спектрах легированных полупроводников при концентрациях свободных носителей заряда $n \sim 10^{16} \mathrm{~cm}^{-3}$ и выше проявляются плазменные колебания электронов проводимости. Плазменная частота $\omega_{p}$ в пренебрежении дисперсией (в пределе $\mathbf{k}=0)$ определяется выражением $\omega_{p}=\left(4 \pi n e^{2} / \varepsilon_{\infty} m^{*}\right)^{1 / 2}$, где $e-$ заряд электрона, $\varepsilon_{\infty}$ - высокочастотная диэлектрическая проницаемость и $m^{*}$ - эффективная масса носителей. Макроскопические электрические поля таких колебаний в полярных полупроводниках взаимодействуют с электрическим полем полярных продольных колебаний решетки. В результате такого взаимодействия образуются связанные плазмон-фононные колебания с перенормированной частотой и соответствующие им коллективные моды проявляются в спектрах рамановского рассеяния.

В исследованиях легированных полупроводников с электронным типом проводимости связанные состояния плазмон-фононных колебаний [10] обычно интерпретировались теоретически в виде двух - низкочастотной $\mathrm{LO}_{-}$и высокочастной $\mathrm{LO}_{+}$ветвей таких связанных состояний, определяемых мнимой частью обратной диэлектрической функции кристалла, зависящей от концентрации $n$.

В отличие от таких традиционно выполняемых исследований, для более строго количественного описания процесса рамановского рассеяния света связанными плазмон-фононными колебаниями, впервые была предсказана необходимость учета в диэлектрической функции кристалла затухания решеточных колебаний $[11,15]$, наряду с затуханием плазменного колебания $\Gamma$, определяемого временем импульсной релаксации электронов проводимости известной формулой $\tau=\mu \varepsilon m^{*} / e$, где $\mu$ - подвижность свободных носителей заряда, $\varepsilon-$ диэлектрическая проницаемость и $m^{*}$ - эффективная масса. Первые результаты по измерению концентраций и подвижностей свободных носителей заряда из данных спектров рамановского рассеяния света связанными плазмон-фононными колебаниями в легированных полупроводниках $n$-GaP в широком диапазоне концентраций и подвижностей свободных носителей заряда были продемонстрированы в работе [15]. Развитый подход, на примере исследований кристаллов $n$ - $\mathrm{GaP}$, позволил создать принципиально новую прецизионную методику рамановской спектроскопии, позволяющей изучать тонкие механизмы электрон-фононного взаимодействия в легированных полупроводниках. Было показано, что обнаружение в спектрах рамановского рассеяния света проявления вкладов новых механизмов резонансных электрон-фононных взаимодействий значительно повышают информативность и надежность данного метода и его аналитические возможности для легированных полупроводников [15-17]. Впервые были получены масштабированные закономерности немонотонных зависимостей спектральных параметров - полуширины и смещения частоты линий связанных плазмон-фононных $\mathrm{LO}(\Gamma)_{+}$колебаний от концентрации $n$ и подвижности $\mu$ свободных носителей заряда. В этой же работе приводятся результаты определения концентраций и подвижностей свободных носителей заряда на основе прямых электрических измерений проводимости и эффекта Холла, выполненные на тех же образцах $n$-GaP. Полученные данные непосредственно указали на их хорошее численное согласие с результатами, полученными по измерению концентраций и подвижностей свободных носителей заряда по спектрам рамановского рассеяния света $\mathrm{LO}(\Gamma)_{+}$колебаниями [15]. На их основе развиты бесконтактные и неразрушающие методы определения совершенства кристаллической структуры и чрезвычайно важных основных электрических параметров полупроводников - концентрации и подвижности носителей заряда. Впоследствии, предложенные развитые подходы для исследования рамановского рассеяния света $\mathrm{LO}(\Gamma)_{+}$колебаниями с определением концентраций $n$ и подвижностей $\mu$ были успешно реализованы в многих мировых научных центрах. Эффективности использования развитых подходов, судя по ссылкам на работы по $n$-GaP [15] и $n$ - $\mathrm{ZnO}$ [16] экспериментально подтверждены опубликованными многочисленными исследованиями (в более чем в 300 случаях) для чрезвычайно перспективных в оптоэлектронике и нанолектронике, в том числе и для современных квантовых технологий, различных полярных легированных полупроводников, таких как: $\mathrm{A}^{2} \mathrm{~B}^{6}$, (например, $\mathrm{ZnO}, \mathrm{ZnS}, \mathrm{ZnSe}, \mathrm{CdS}$, $\mathrm{CdTe}), \mathrm{A}^{3} \mathrm{~B}^{5}(\mathrm{GaN}, \mathrm{GaAs}, \mathrm{InP}, \mathrm{InN}), \mathrm{A}^{4} \mathrm{~B}^{4}$ (4H-SiC, $6 \mathrm{H}-\mathrm{SiC}, \beta-\mathrm{SiC})$ и многими другими. В то же время, судя по ссылкам на последующие обзоры, только по $\mathrm{GaN}$ [18], $\mathrm{SiC}$ [19], InP [20] и другими, основывающимся на результатах использования подходов, разработанных в оригинальных работах $[15,16]$, число использований и подтверждений, введенных в $[15,16]$ подходов, существенно превышает 1000, став, таким образом, принципиальной основой современной оптической метрологии полупроводниковых материалов. 
В настоящей работе показано, что на основе развития спектроскопии микрорамановского рассеяния света может быть реализован метод исследования совершенства кристаллической структуры при одновременном бесконтактном неразрушающем измерении локальной концентрации носителей заряда в гомоэпитаксиальных слоях наномасштабной толщины. Такие исследования выполнены на примере монокристалла кристалла (001) $n$-GaP толщиной $70 \mathrm{~nm}$, выращенного методом газофазной эпитаксии из металлоорганических соединений на проводящей сильнолегированной подложке кристалла $n$-GaP, ориентированного по оси (001).

\section{2. Методика эксперимента}

Для наших исследований использовался образец $n$-GaP/n-GaP (001) со специально нелегированным наномасштабным гомоэпитаксиальным слоем $n$-GaP толщиной $70 \mathrm{~nm}$, выращенным методом газофазной эпитаксии из металлоорганических соединений на проводящей сильнолегированной подложке кристалла $n$ - $\mathrm{GaP}$, ориентированного по оси (001) (образец № 1). Для непосредственного сравнения использовался образец № 2 сильно компесированного высокоомного (с удельным сопротивлением $\sim 10^{12} \Omega \cdot \mathrm{cm}^{-1}$ ) полуизолирующего кристалла $s i$-GaP, также ориентированного по оси (001).

Возбуждение спектров микрорамановского рассеяния света осуществлялось излучением второй гармоники непрерывного лазера на алюмоиттриевом гранате с длиной волны $\lambda_{i}=532.070 \mathrm{~nm}$ по методике, приведенной в $[25,26]$. Спектральный состав рассеянного света анализировался с помощью конфокального рамановского спектрометра HR 800 (Horiba Jobin Yvon), оснащенного микроскопом Olympus BX 41 с микрообъективом 100× (NA 0.9). Локальный размер возбуждающего пятна составлял $\sim 0.9 \mathrm{mkm}$. Измерения спектров рассеяния света выполнены в одних и тех же экспериментальных условиях для всех образцов при комнатной температуре и в конфигурации обратного рассеяния при параллельной поляризации падающего и рассеянного света $Z(X X) Z$ с осями $X, Y$ и $Z$, соответственно, вдоль направлений $[11 \overline{0}],[110]$ и [001].

\section{3. Экспериментальные результаты исследований и их обсуждение}

Как уже отмечалось выше, одной из основных проблем измерения структурных и электрических параметров гомоэпитаксиальных слоев, выращенных на сильнолегированных подложках являются очевидные сложности определения концентрации и подвижности свободных носителей заряда на основе традиционного метода исследования электрических свойств полупроводниковых материалов путем выполнения электрических измерений проводимости и эффекта Холла [12]. В особенности, такие сложности возникают для гомоэпитаксиальным слоев наномасштабной толщины.

На рисунке представлены типичные фрагменты спектров рамановского рассеяния света, измеренные в конфигурации обратного рассеяния при параллельной поляризации и рассеянного света $Z(X X) Z$ - в диапазоне частот $350-450 \mathrm{~cm}^{-1}$ для гомоэпитаксиального слоя $n$-GaP (кривая с точками) и сильнолегированной подложки $n$-GaP (001) (кривая сточками) в исследуемом образце № 1 - -GaP/n-GaP (001) в сравнении со спектром для образца № 2 полуизолирующего кристалла $s i$-GaP (001) (кривая с кружками). Толщина гомоэпитаксиального слоя, выращенного методом газофазной эпитаксии из металлоорганических соединений на проводящей подложке $n$-GaP, ориентированной по оси (100), равна $70 \mathrm{~nm}$. Все спектры получены в одних и тех же экспериментальных условиях при комнатной температуре и при возбуждении излучением лазера с длиной волны $\lambda_{i}=532.070 \mathrm{~nm}$ с одинаковой плотностью мощности лазерного излучения. Указанные на спектрах численные величины значения частоты и истинные полуширины линии - полная ширина на половине ее высоты $\left(\mathrm{B} \mathrm{cm}^{-1}\right)$ получены с выделением лоренцевского контура из наблюдаемого фохтовского контура с учетом поправки на спектральное разрешение, описываемое гаусовским контуром.

Из приведенных на рисунке спектров хорошо видно, что в рамановском спектре, выявленном для гомоэпитаксиального слоя $n$-GaP, толщиной $70 \mathrm{~nm}$ также удается обнаружить две достаточно узкие характерные линии, типичные для других зарегистрированных спектров и для сильнолегированной подложки $n$ - $\mathrm{GaP}(001)$ и полуизолирующего кристалла $s i$-GaP (001). Помимо этого также хорошо заметно, что все низкочастотные линии при $365.50 \mathrm{~cm}^{-1}$ имеют одинаковые численные значения интенсивностей, частот и полуширин. В то же время, все совсем, наоборот, для высокочастотных линий. Они существенно отличаются между собой по спектральным параметрам и имеют разные численные значения интенсивностей, частот и полуширин. В высокоомном (с удельным сопротивлением $\sim 10^{12} \Omega \cdot \mathrm{cm}^{-1}$ ) полуизолирующем кристалле $s i$-GaP (001) концентрация свободных носителей заряда $\mathrm{n}$ меньше чем $10^{17} \mathrm{~cm}^{-3}$ и, соответственно, плазменным вкладом свободных носителей можно пренебречь. Поэтому наблюдаемые типичные линии можно интерпретировать как обусловленные колебаниями поперечных ТО(Г) - (при $365.50 \mathrm{~cm}^{-1}$ с полушириной $3.45 \mathrm{~cm}^{-1}$ ) и продольных $\mathrm{LO}(\Gamma)$ (при $402.30 \mathrm{~cm}^{-1}$ с полушириной $0.60 \mathrm{~cm}^{-1}$ ) оптических фононов.

Соответственно, характерные смещенные линии, в спектрах гомоэпитаксиальныого слоя $n-\mathrm{GaP}$, толщиной $70 \mathrm{~nm}$ и для сильнолегированной подложки $n-\mathrm{GaP}(001)$, в исследуемом образце № $1 n$-GaP/n-GaP (001), могут быть интерпретированы как соответствующие связанным высокочастотным продольным плазмон-фононным 


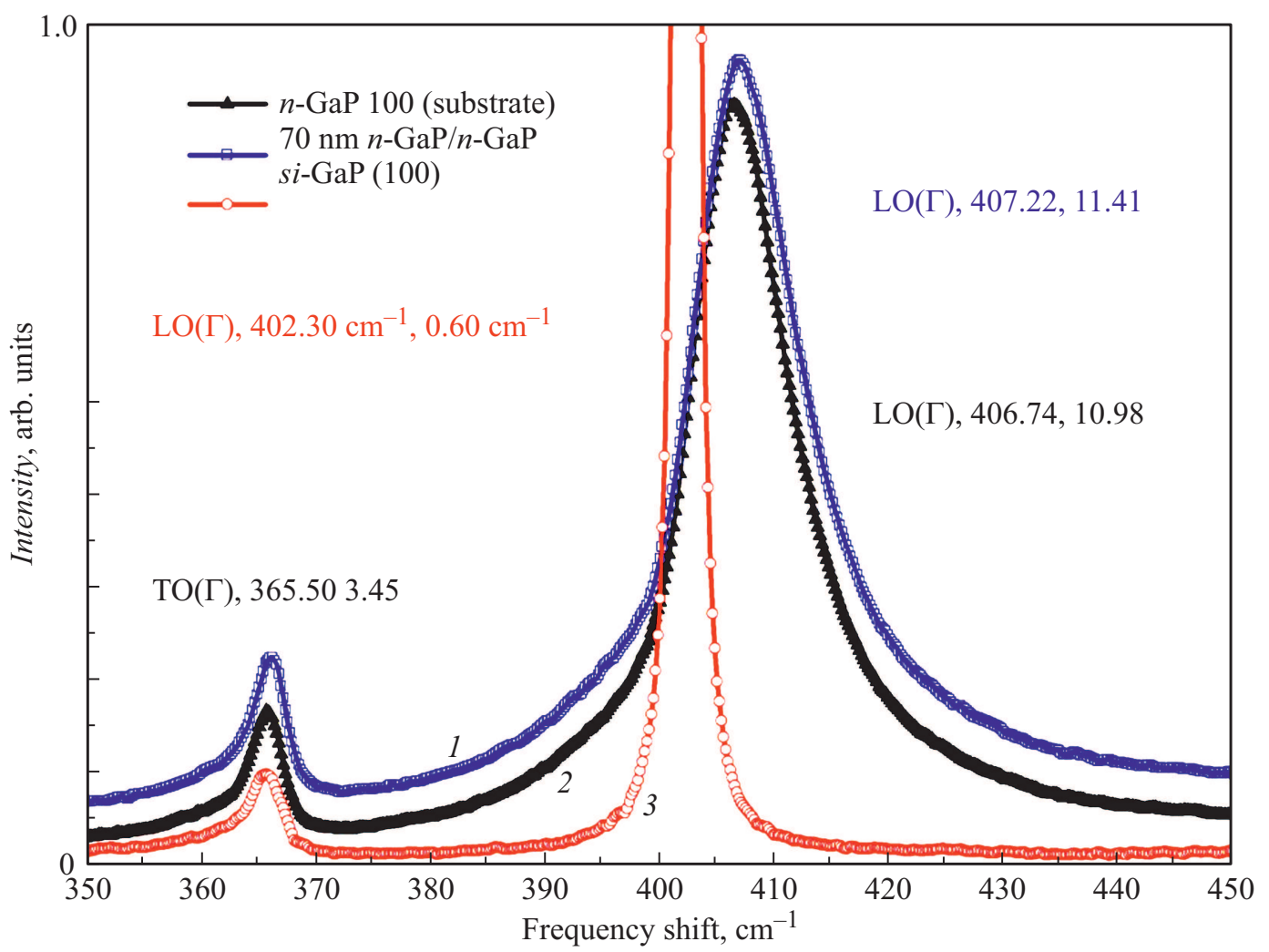

Спекры рамановского рассеяния света поперечными $\mathrm{TO}(\Gamma)_{-}$и продольными $\mathrm{LO}(\Gamma)$ оптическими фононами, а также высокочастотными продольными связанными плазмон-фононными $\mathrm{LO}(\Gamma)_{+}$колебаниями в диапазоне частот $350-450 \mathrm{~cm}^{-1}$ в кристаллах (001) si-GP (кривая 3), (001) $n$-GaP (кривая 2) и образце $n$-GaP/n-GaP с гомоэпитаксиальным нанослоем $n$-GaP (кривая 1). Спекры усиленные по вертикальной шкале интенсивности рассеяния, четко демонстрируют, эволюцию спектральных параметров. Спектры получены при комнатной температуре и при возбуждении излучением лазера с длиной волны $\lambda_{i}=532.070 \mathrm{~nm}$. Толщина нанослоя, выращенного методом газофазной эпитаксии из металлоорганических соединений на проводящей подложке $n$-GaP, ориентированной по оси $(001)$, равна $70 \mathrm{~nm}$. Указанные величины значения частоты и полуширин линии (полных ширин на половине интенсивости) полученных с выделением лоренцевого контура из наблюдаемого фохтовского контура с учетом поправки на спектральное разрешение. Помимо этого, приведены результаты численного расчета для концентрации $n$ и подвижности $\mu$ свободных носителей заряда гомоэпитаксиального слоя наномасштабной толщины и проводящей сильнолегированной подложки. Расчеты выполнены на основе микроскопической модели рассеяния света $\mathrm{LO}(\Gamma)_{+}$колебаниями, обусловленного механизмами деформационного потенциала и электрооптическим рассеянием. Рисунки в цвете online.

$\mathrm{LO}(\Gamma)_{+}$колебаниям. В полярных кристаллах образование таких связанных электрон-фононных состояний обусловлено механизмами деформационного потенциала и электрооптическим рассеянием [12]. Необходимо отметить, что колебания поперечных $\mathrm{TO}(\Gamma)_{-}$фононов не образуют связанных плазмон-фононных состояний [12] и, как отмечалось выше, непосредственно измеренные значения частот и ширин линий не изменялись. Обнаружение и четкое выявление спектров продольных плазмон-фононных $\mathrm{LO}(\Gamma)_{+}$колебаний при $407.22 \mathrm{~cm}^{-1}$ с полушириной $11.41 \mathrm{~cm}^{-1}$ для гомоэпитаксиальныого слоя $n$-GaP (001) и, соответственно, $406.74 \mathrm{~cm}^{-1}$ с полушириной $10.98 \mathrm{~cm}^{-1}$ для подложки $n$-GaP (001) позволяет бесконтактным и неразрушающим образом определять локальную концентрацию $n$ и подвижность $\mu$ свободных носителей заряда для гомоэпитаксиальныого наномасштабного слоя $n$-GaP, толщиной $70 \mathrm{~nm}$ и выращенного на проводящей сильнолегированной подложки $n$-GaP (001). Расчеты выполнены на основе микроскопической модели рассеяния света $\mathrm{LO}(\Gamma)_{+}$колебаниями, обусловленного механизмами деформационного потенциала и электрооптическим рассеянием [12]. Полученные численные значения оказались равными $n_{\text {hepi }}=(3.25 \pm 0.1) \cdot 10^{17} \mathrm{~cm}^{-3}$ и подвижности $\quad \mu_{\text {hepi }}=(40.0 \pm 0.1) \mathrm{cm}^{2} \cdot \mathrm{V}^{-1} \cdot \mathrm{s}^{-1}$ для гомоэпитаксиального слоя (100) $n$-GaP в сравнении с $n_{\text {subs }}=(2.52 \pm 0.1) \cdot 10^{17} \mathrm{~cm}^{-3}$ и $\mu_{\text {subs }}=$ $=(51.0 \pm 0.1) \mathrm{cm}^{2} \cdot \mathrm{V}^{-1} \cdot \mathrm{s}^{-1}$ подложки (100) $n$-GaP образца $n-\mathrm{GaP} / n-\mathrm{GaP}(100)$.

Как видно, концентрации и подвижности носителей заряда в наномасштабном гомоэпитаксиальном слое (001) $n$-GaP и объемной подложке (001) $n$-GaP оказались близкими по порядку величины. Несмотря на казалось бы очень близкие значения величин частот линий плазмонфононных $\mathrm{LO}(\Gamma)_{+}$колебаний и на небольшую разницу концентраций $\Delta n_{\text {hepi }}=(0.73 \pm 0.1) \cdot 10^{17} \mathrm{~cm}^{-3}$ для гомо- 
эпитаксиального слоя (001) n-GaP и подложки (001) $n$-GaP, соответствующие сигналы в довольно узком частотном диапазоне достаточно хорошо разрешены и четко прослеживаются в спектральных измерениях.

\section{4. Заключение}

В работе представлены результаты исследования методом КРС структурных и электрических свойств гомоэпитаксиального нанослоя $n-\mathrm{GaP}$, толщиной $70 \mathrm{~nm}$, выращенного газофазной эпитаксией из металлоорганических соединений на проводящей сильнолегированной подложке кристалла $n$-GaP, ориентированного по оси (001). Показано, что в спектре рамановского рассеяния света такого нанослоя $n-\mathrm{GaP}$ в образце $n-\mathrm{GaP} / n-\mathrm{GaP}$ (001) в сравнении со спектром высокоомного кристаллического образца (001) si-GaP удается обнаружить две достаточно узкие линии. Они приписаны колебаниям поперечных $\mathrm{TO}(\Gamma)$ фононов и высокочастотным продольным связанным плазмон-фононным $\mathrm{LO}(\Gamma)_{+}$колебаниям. На основании таких экспериментальных данных также установлено, что спектральные параметры $\mathrm{LO}(\Gamma)_{+}$колебаний как в нанослое $n$-GaP, так и в подложке $(001)$ $n$-GaP существенно отличаются друг от друга и от спектральных параметров линии $\mathrm{LO}(\Gamma)$ продольных оптических фононов в si-GaP. Анализ выявленных строгих количественных особенностей спектральных параметров позволил получить ценную информацию о совершенстве кристаллической структуры наномасштабного эпитаксиального слоя (001) n-GaP. Помимо этого, показано что, численные расчеты на основе микроскопической модели рассеяния света $\mathrm{LO}(\Gamma)_{+}$колебаниями, обусловленного механизмами деформационного потенциала и электрооптическим рассеянием, позволяют бесконтактным и неразрушающим образом определить концентрацию и подвижность свободных носителей заряда гомоэпитаксиального слоя наномасштабной толщины. Полученные значения концентрации носителей заряда оказались $\quad n_{\mathrm{hep}} i=(3.25 \pm 0.1) \cdot 10^{17} \mathrm{~cm}^{-3} \quad$ и $\mu_{\mathrm{hepi}}=$ $=(40.0 \pm 0.1) \mathrm{cm}^{2} \cdot \mathrm{V}^{-1} \cdot \mathrm{s}^{-1}$ для гомоэпитаксиального слоя (001) $n$-GaP в сравнении с $\quad n_{\text {subs }}=(2.52 \pm 0.1) \cdot 10^{17} \mathrm{~cm}^{-3} \quad$ и $\quad \mu_{\text {subs }}=$ $=(51.0 \pm 0.1) \mathrm{cm}^{2} \cdot \mathrm{V}^{-1} \cdot \mathrm{s}^{-1}$ для подложки $(001)$ $n-\mathrm{GaP}$ образца $n-\mathrm{GaP} / n-\mathrm{GaP}(001)$.

\section{Конфликт интересов}

Авторы заявляют, что у них нет конфликта интересов.

\section{Список литературы}

[1] M. Itoh. Prog. Surf. Sci. 66, 53 (2001).

[2] P. Kratzer, C.G. Morgan, M. Scheffler. Phys. Rev. B 59, 15246 (1999).

[3] Ю.Г. Галицын, Д.В. Дмитриев, В.Г. Мансуров, С.П. Мощенко, А.И. Торопов. Письма в ЖЭТФ 86, 553 (2007).
[4] O.A. Ageev, M. Solodovnik, S.V. Balakirev, I.A. Mikhalin, M. Eremenko. J. Crystal Growth 457, 46 (2017).

[5] О.С. Комков, А.Н. Пихтин, Ю.В. Жиляев, Л.М. Федоров. Письма в ЖТФ 34, 1, 81 (2008).

[6] B. Németh, W. Kunert, K. Stolz, K. Volz. J. Cryst. Growth 310, 1595 (2008).

[7] S. Nagarajan, H. Jussila, J. Lemettinen, K. Banerjee, M. Sopanen, H. Lipsanen. J. Phys. D 46, 165103 (2013).

[8] О.С. Комков, Д.Д. Фирсов, Т.В. Львова, И.В. Седова, А.Н. Семенов, В.А. Соловьев, С.В. Иванов. ФТТ 58, 707 (2016).

[9] K. Storm, F. Halvardsson, M. Heurlin, D. Lingren, A. Gustafsson, P.M. Wu, B. Monemar, L. Samuelson. Nature Nanotechnol. 7, 718 (2012).

[10] Light Scattering in Solids, Topics in Applied Physics / Ed. M. Cardona, G. Guntherodt. Springer, Berlin, Heidelberg, N.Y. (1974). 543 p.

[11] B.H. Bairamov, V.A. Voitenko, V.V. Toporov, G. Irmer, J. Monecke. Phys. Status Solidi 1, 2773 (2004).

[12] F.H. Bayramov, G. Irmer, V.V. Toporov, B.H. Bairamov. Jpn. J. Appl. Phys. 50, 05FE06 (2011).

[13] Б.Х. Байрамов, В.А. Войтенко, И.П. Ипатова. УФН 163, 67 (1993).

[14] B.H. Bairamov, V.A. Voitenko, I.P. Ipatova. Phys. Rep. 229, 221 (1993).

[15] B.H. Bairamov, I.P. Ipatova, V.V. Toporov, V.A. Voitenko, G. Irmer, J. Monecke, E. Jahne. Appl. Surf. Sci. 50, 1, 300 (1991).

[16] B.H. Bairamov, V.A. Voitenko, B.P. Zakharchenya, V.V. Toporov, M. Henini, A.J. Kent. Nanotechnol. 11, 314 (2000).

[17] G. Irmer, V.V. Toporov, B.H. Bairamov, J. Monecke. Phys. Status Solidi 119, 2, 595 (1983).

[18] B.H. Bairamov A. Heinrich, G. Irmer, V.V. Toporov, E. Ziegler. Phys. Status Solidi B 119, 1, 227 (1983).

[19] J.T. Holmi, B.H. Bairamov, S. Suihkonen, H. Lipsanen. J. Cryst. Growth 499, 47 (2018).

[20] H. Harima. J.Condens. Matter. Phys. 14, 38, 967 (2002).

[21] S. Nakashima, H. Harima. Phys. Status Solidi 162, 39 (1997).

[22] L. Artús, R. Cuscó, J. Ibáñez, N. Blanco, G. González-Díaz. Phys. Rev. B 60, 5456 (1999).

[23] B.H. Bairamov, V.V. Toporov, F.B. Bayramov. Semicond. 53, 2129 (2019).

[24] Б.Х. Байрамов, В.В, Топоров, Ф.Б. Байрамов. ФТП 54, 1189 (2020).

[25] Ф.Б. Байрамов, В.В, Топоров, О.Б. Чакчир, В.Н. Анисимов, Б.Х. Байрамов. Письма в ЖТФ 44, 12, 3 (2018).

[26] Б.Х. Байрамов. ФТТ 58, 707 (2016).

Редактор К.В. Емцев 